\title{
Lifestyle and dietary habits of patients with gout followed in rheumatology settings
}

\author{
M. Manara ${ }^{1,2}$, G. Carrara ${ }^{2}$, C.A. Scirè2, M.A. Cimmino 3 , M. Govoni ${ }^{4}$, \\ C. Montecucco ${ }^{5}$, M. Matucci-Cerinic ${ }^{6}$, G. Minisola ${ }^{7}$ and the KING study group \\ ${ }^{1}$ Gaetano Pini Institute, Milano, Italy; \\ Epidemiology Unit, Italian Society for Rheumatology, Milano, Italy; \\ ${ }^{3}$ Research Laboratory and Academic Unit of Clinical Rheumatology, Department of Internal Medicine, \\ University of Genoa, Italy; \\ ${ }^{4}$ Department of Medical Sciences, University of Ferrara, Italy; \\ ${ }^{5}$ Division of Rheumatology, IRCCS San Matteo Foundation, Pavia, Italy; \\ ${ }^{6}$ Department of Internal Medicine, University of Florence, Italy; \\ ${ }^{7}$ Department of Rheumatology, San Camillo Hospital, Rome, Italy
}

\section{SUMMARY}

Diet and lifestyles modification are core aspects of the non-pharmacological management of gout, but a poor consistency with suggested guidelines is reported. This study aimed to investigate dietary and lifestyle habits of patients with gout followed in rheumatology settings.

Data were retrieved from the baseline dataset of the KING study, a multicentre cohort study of patients with gout followed in rheumatology settings. Dietary habits were assessed with the Italian National Institute of Statistics (ISTAT) food-frequency questionnaire and compared with reported data about general population. The relative increase of exposure was estimated by standardized prevalence ratios adjusted for gender, age and geographical distribution.

The study population included 446 patients, with a mean age of 63.9 years and a $\mathrm{M} / \mathrm{F}$ ratio of 9:1. Compared to the Italian population, gouty patients showed a higher prevalence of obesity [1.82 (1.52-2.18)] and a higher consumption of wine [1.85 (1.48-2.32)] and beer [2.21 (1.68-2.90)], but a lower prevalence of smoking and a lower intake of liquor. They showed a lower intake of red meat [0.80 (0.71-0.91)], but a similar intake of other tested dietary factors.

Gouty patients' lifestyle is still partially different from the recommended.

Key words: Gout; diet; lifestyle.

Reumatismo, 2015; 67 (4): 138-148

\section{INTRODUCTION}

out is the most common inflammatory arthritis in men and its prevalence has been increasing in the last years due to progressive aging of the population, increased use of drug, and changes in lifestyles and dietary habits (1).

Dietary and lifestyle factors have been reported to be implicated in the manifestation of gout, being associated with an increased risk of hyperuricemia and incident gout in general population (2). Many of these risk factors have been also identified as triggers for acute flares in patients with prevalent gout (2). Based on these findings, international recommendations for the management of gout recognise dietary intervention and lifestyle modification as core aspects of the non-pharmacological management of gout $(3,4)$.

Nevertheless, since pharmacological management and drug adherence are largely suboptimal (5), we expect dietary habits very different from those recommended for gout. A recent study investigated dietary habits of a small sample of patients with gout, followed in a rheumatologic setting, and it has provided preliminary evidence of poor consistency with suggested guidelines (6).

Therefore, the aim of the present study was to obtain relative measures of exposure to dietary and lifestyle risk factors in patients with prevalent gout followed in rheumatologic settings compared with general population, in order to identify needs for intervention in these patients. For this purpose, a random sample of Italian patients with gout 
from rheumatology centres was drawn and lifestyles and dietary habits were compared with official data from general population.

\section{MATERIALS AND METHODS}

A cross-sectional analysis of data retrieved from the baseline dataset of the KING study (Kick-off of the Italian Network for Gout, NCT01549210) was done. The KING study is a multicentre cohort study including patients with a previous clinical diagnosis of gout formulated by rheumatologists. Patients were recruited between June 2011 and January 2012 from 30 rheumatology centres across Italy. Participants were selected from clinical registries of all patients with gout evaluated in the previous two years in each centre by random cluster sampling.

A rheumatologist interviewed patients about demographics, level of education and employment status. Dietary habits were assessed using the food-frequency questionnaire created by the Italian National Institute of Statistics (ISTAT) for national surveys (www.istat.it/it/archivio/14562).

Data about the Italian population were collected from the last available national survey reported by ISTAT in the 2010 release of the Health for All, stratified for the maximum level of detail in terms of gender, classes of age and geographic region (7).

\section{Statistical analysis}

Lifestyle and dietary variables were categorized according to ISTAT definitions or, for dietary factors, to cut-offs reported to be significantly associated with an increased risk of gout. Obesity was defined as a body mass index (BMI) $\geq 30$. Smoking was considered for current smokers, while ex-smokers were assessed as non-smokers. The cut-off for a high wine consumption was established at $\geq 0.5$ litres per day, while a high beer consumption was defined for usual drinkers (daily consumers). Liquor consumption was defined for any amount of spirits consumed. The intake of beef meat, sausages, pork meat and fish was considered high when subjects consumed these foods more than once a week, while a high intake of carbohydrates, milk, dairy products, fruit or vegetables was defined for $\geq 1$ servings per day. Employment rate was defined as the percentage of employed subjects in the reference population aged 15 and older. A low educational level was assigned to illiterates and subjects who got only a primary school licence.

The relative increase of exposure in gouty patients was estimated by standardized prevalence ratios (SPR), calculated as the ratio between the observed number of exposed subjects and the expected number, based on data derived from the reference population, stratifying by gender, age and geographical distribution (8). For each variable, confounding was systematically explored by stratification for principal comorbidities, which could explain lifestyle or dietary changes in gouty patients. Analyses were performed using STATA (Stata Corp, 2009, release 11, TX, USA).

\section{RESULTS}

The analysis includes 446 patients, with a median disease duration of 3.7 years, higher prevalence of male subjects $(90.4 \%)$, mean

Table I - Characteristics of the study population $(n=446)$.

\begin{tabular}{|l|l|}
\hline Age [mean (SD)] & $63.9(11.6)$ \\
\hline Gender (male) [number (\%)] & $403(90.36 \%)$ \\
\hline BMI [mean (SD)] & $28.0(4.07)$ \\
\hline Disease duration (years) [median (IQR)] & $3.7(1.4-10.2)$ \\
\hline Joint involvement [number (\%)]: & \\
- Monoarticular & $97(21.95 \%)$ \\
- Oligoarticular & $262(59.28 \%)$ \\
- Polyarticular & $83(18.78 \%)$ \\
\hline Tophaceous gout [number (\%)] & $87(19.91 \%)$ \\
\hline Serum urate level (mg/dL) [mean (SD)] & $6.3(1.75)$ \\
\hline Allopurinol [number (\%)] & $304(68.16 \%)$ \\
\hline Febuxostat [number (\%)] & $59(13.23 \%)$ \\
\hline Comorbidities [number (\%)]: & \\
- Hypertension & $306(68.76 \%)$ \\
- Diabetes & $62(13.90 \%)$ \\
- Dyslipidemia & $184(41.91 \%)$ \\
- Ischemic heart disease & $58(13.09 \%)$ \\
- Cerebral vascular disease & $24(5.39 \%)$ \\
- Nephrolithiasis & $96(21.52 \%)$ \\
- Renal failure & $54(12.16 \%)$ \\
\hline
\end{tabular}

$\mathrm{SD}$, standard deviation; BMI, body mass index; IQR, interquartile range. 
Table II - Observed and expected exposure to dietary and lifestyle factors in the study population, with standardized prevalence ratios $(n=446)$.

\begin{tabular}{|c|c|c|c|}
\hline & $\begin{array}{c}\text { Observed } \\
\mathrm{N}(\%)\end{array}$ & $\begin{array}{c}\text { Expected } \\
\mathrm{N}(\%)\end{array}$ & SPR (95\% Cl) \\
\hline Obesity (BMI $\geq 30$ ) & $114(25.56 \%)$ & $63(14.12 \%)$ & $1.82(1.52-2.18)$ \\
\hline Smoking (current smokers) & $72(16.22 \%)$ & $96(21.52 \%)$ & $0.75(0.60-0.95)$ \\
\hline Wine ( $\geq 0.5$ l/day) & $73(16.52 \%)$ & $39(8.74 \%)$ & $1.85(1.48-2.32)$ \\
\hline Beer (daily consumption) & $49(11.06 \%)$ & $22(4.93 \%)$ & $2.21(1.68-2.90)$ \\
\hline Spirit (any amount) & $72(16.25 \%)$ & $125(28.03 \%)$ & $0.57(0.48-0.72)$ \\
\hline Beef meat ( $\geq 1$ serving/week) & $249(56.21 \%)$ & $313(70.18 \%)$ & $0.80(0.71-0.91)$ \\
\hline Sausages ( $\geq 1$ serving/week) & $278(62.61 \%)$ & $264(59.19 \%)$ & $1.05(0.94-1.18)$ \\
\hline Pork meat ( $\geq 1$ serving/week) & $158(35.75 \%)$ & $206(46.19 \%)$ & $0.77(0.66-0.90)$ \\
\hline Fish ( $\geq 1$ serving/week) & 264 (59.50\%) & $267(59.86 \%)$ & $0.98(0.87-1.11)$ \\
\hline Carbohydrates ( $\geq 1$ serving/day) & $391(87.67 \%)$ & $389(87.22 \%)$ & $1.00(0.91-1.11)$ \\
\hline Milk ( $\geq 1$ serving /day) & $194(43.60 \%)$ & $238(53.36 \%)$ & $0.81(0.71-0.94)$ \\
\hline Dairy products ( $\geq 1$ serving/day) & $109(24.60 \%)$ & $108(24.21 \%)$ & $1.01(0.83-1.21)$ \\
\hline Vegetables ( $\geq 1$ serving/day) & $249(56.33 \%)$ & $251(56.28 \%)$ & $0.99(0.87-1.12)$ \\
\hline Fruit ( $\geq 1$ serving/day) & $366(82.25 \%)$ & 366 (82.25\%) & $1.00(0.90-1.11)$ \\
\hline Employment rate & $137(32.54 \%)$ & $278(62.33 \%)$ & $0.47(0.40-0.56)$ \\
\hline Illiteracy or primary school education & $147(32.96 \%)$ & $86(19.28 \%)$ & $1.71(1.45-2.00)$ \\
\hline
\end{tabular}

SPR, standardized prevalence ratios; $\mathrm{Cl}$, confidence interval; $\mathrm{BMI}$, body mass index.

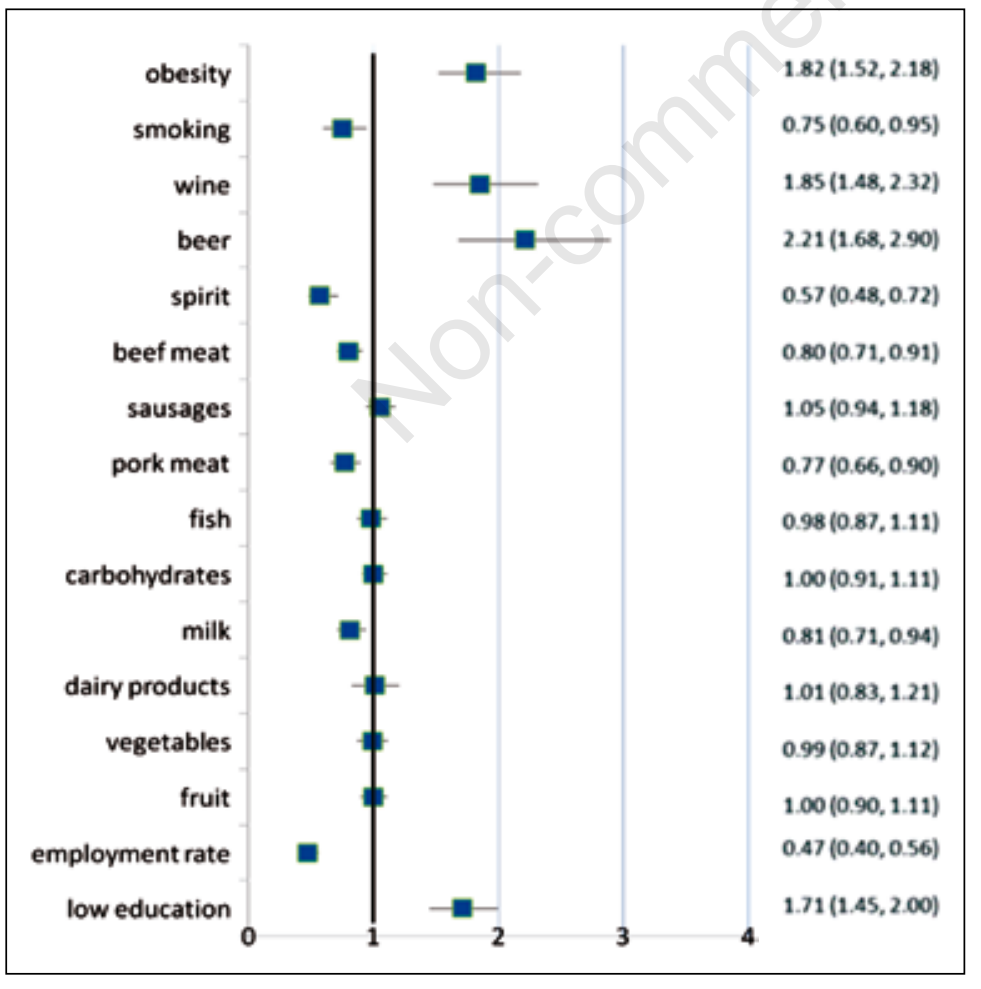

Figure 1 - Relative exposure to lifestyle and dietary risk factors in gouty patients compared to the general population [standardized prevalence ratios, SPR (95\% confidence intervals)]. age of 63.9 years and an average BMI of 28 . Physical examination revealed the presence of tophi in almost $20 \%$ of patients and a polyarticular involvement in $83(18.8 \%)$ subjects. Although most of patients where on uratelowering treatment $(81.4 \%)$, mean serum uric acid was above recommended levels $(6.3 \mathrm{mg} /$ dL). A high proportion of subjects reported comorbidities, especially in the spectrum of the metabolic syndrome (Table I).

Compared to the Italian population, gouty patients showed a higher prevalence of obesity (SPR: 1.82, 95\%CI: 1.52-2.18) (Table II and Figure 1). Analysis of lifestyle habits revealed a slightly lower prevalence of smoking and a lower consumption of spirits, but a significant higher consumption of wine (SPR: 1.85, 95\%CI: 1.48-2.32) and beer (SPR: 2.21, 95\%CI: 1.68-2.90) in patients with gout. By considering purine-rich food consumption, gouty patients showed a lower intake of beef meat (SPR: 0.80, 95\%CI: 0.71-0.91) and pork meat (SPR: 0.77, 95\%CI: 0.66-0.90), but a similar amount of sausages and fish than Italian general population. Among other tested dietary factors, we observed a lower con- 
sumption of milk but no significant differences in the amount of dairy products, carbohydrates, vegetables and fruit consumed. In the end, we found that gouty patients had a lower employment rate and a lower educational level compared to the Italian population, suggesting a lower socio-economic status.

Results from stratified analyses are reported as supplementary material (Appendix 1).

\section{DISCUSSION}

This survey on lifestyle and dietary habits of a large random sample of patients with gout followed in rheumatology practices confirmed previous reported data about unhealthy lifestyles in gouty patients.

The cross-sectional design of the study did not allow us to establish a causal relationship between lifestyle habits and gout occurrence, but we could only verify if gouty patients had current habits consistent with optimal lifestyles. Furthermore, we could not assess if patients had modified their lifestyles and dietary behavior after the diagnosis.

Dietary habits were measured with the same instrument for the studied population and the reference population, and this is the principal strength of our study, leading to an optimal comparability of the two groups. Nevertheless, some dietary factors which have been reported to influence the risk of gout were not included in the ISTAT food-frequency questionnaire.

Weight loss is a key aspect of the non-pharmacological management of gout (3). A high prevalence of overweight and obesity in patients with gout has been reported in several cross-sectional studies, and obesity was associated with an increased risk of incident gout in prospective studies $(9,10)$. Gouty patients included in our study population showed a higher prevalence of obesity compared to the Italian general population. Moreover, the prevalence of obesity was even higher in patients affected by heart

\section{APPENDIX 1: SUPPLEMENTARY DATA}

Standardized prevalence ratios (SPR) of observed and expected exposure to dietary and lifestyle factors in the study population stratified for principal comorbidities.

\section{Obesity}

\begin{tabular}{|l|c|c|c|}
\hline & $\mathbf{n .}$ & SPR & 95\% IC \\
\hline Non-stratified & 446 & 1.82 & $1.52-2.18$ \\
\hline Ischemic heart disease (yes) & 58 & 2.38 & $1.56-3.65$ \\
Ischemic heart disease (no) & 388 & 1.76 & $1.44-2.14$ \\
\hline Diabetes (yes) & 62 & 2.32 & $1.53-3.52$ \\
Diabetes (no) & 384 & 1.73 & $1.42-2.12$ \\
\hline Cerebral vascular & 24 & 1.34 & $0.56-3.20$ \\
disease (yes) & & & \\
Cerebral vascular & 422 & 1.85 & $1.53-2.22$ \\
disease (no) & & & \\
\hline Dyslipidemia (yes) & 184 & 1.84 & $1.40-2.42$ \\
Dyslipidemia (no) & 262 & 1.84 & $1.46-2.33$ \\
\hline Renal failure (yes) & 54 & 1.45 & $0.83-2.55$ \\
Renal failure (no) & 392 & 1.88 & $1.56-2.28$ \\
\hline ASA (yes) & 104 & 2.52 & $1.85-3.43$ \\
ASA (no) & 342 & 1.69 & $1.36-2.08$ \\
\hline Anticoagulant (yes) & 44 & 1.97 & $1.13-3.43$ \\
Anticoagulant (no) & 402 & 1.86 & $1.55-2.24$ \\
\hline Comorbidities $\geq 3$ & 231 & 1.93 & $1.52-2.45$ \\
Comorbidities <3 & 215 & 1.69 & $1.28-2.23$ \\
\hline
\end{tabular}

\section{Smoking}

\begin{tabular}{|l|c|c|c|}
\hline & $\mathbf{n}$ & SPR & 95\% IC \\
\hline Non-stratified & 446 & 0.75 & $0.60-0.95$ \\
\hline Ischemic heart disease (yes) & 58 & 0.56 & $0.25-1.23$ \\
Ischemic heart disease (no) & 388 & 0.77 & $0.60-0.97$ \\
\hline Obesity (yes) & 114 & 0.61 & $0.37-1.01$ \\
Obesity (no) & 332 & 0.78 & $0.60-1.01$ \\
\hline Diabetes (yes) & 62 & 0.84 & $0.45-1.55$ \\
Diabetes (no) & 384 & 0.74 & $0.58-0.95$ \\
\hline Cerebral vascular & 24 & 0.85 & $0.31-2.26$ \\
disease (yes) & 422 & 0.74 & $0.59-0.94$ \\
Cerebral vascular & & & \\
disease (no) & 184 & 0.75 & $0.53-1.08$ \\
\hline Dyslipidemia (yes) & 262 & 0.75 & $0.55-1.00$ \\
Dyslipidemia (no) & 54 & 0.80 & $0.40-1.60$ \\
\hline Renal failure (yes) & 392 & 0.74 & $0.58-0.94$ \\
Renal failure (no) & 104 & 0.47 & $0.25-0.88$ \\
\hline ASA (yes) & 342 & 0.78 & $0.61-1.00$ \\
\hline ASA (no) & 44 & 0.35 & $0.12-1.02$ \\
\hline Anticoagulant (yes) & 402 & 0.77 & $0.61-0.97$ \\
Anticoagulant (no) & 231 & 0.69 & $0.48-0.98$ \\
\hline Comorbidities $\geq 3$ & 215 & 0.80 & $0.59-1.08$ \\
Comorbidities <3 & & &
\end{tabular}


Beef consumption

\begin{tabular}{|l|c|c|c|}
\hline & $\mathbf{n}$ & SPR & 95\% IC \\
\hline Non-stratified & 446 & 0.80 & $0.70-0.90$ \\
\hline Ischemic heart disease (yes) & 58 & 0.89 & $0.64-1.23$ \\
Ischemic heart disease (no) & 388 & 0.78 & $0.69-0.89$ \\
\hline Obesity (yes) & 114 & 0.80 & $0.62-1.04$ \\
Obesity (no) & 332 & 0.79 & $0.69-0.91$ \\
\hline Diabetes (yes) & 62 & 0.90 & $0.66-1.23$ \\
Diabetes (no) & 384 & 0.78 & $0.68-0.89$ \\
\hline Cerebral vascular & 24 & 0.69 & $0.40-1.22$ \\
disease (yes) & 422 & 0.80 & $0.71-0.91$ \\
Cerebral vascular & & & \\
disease (no) & 184 & 0.76 & $0.63-0.92$ \\
\hline Dyslipidemia (yes) & 262 & 0.83 & $0.71-0.97$ \\
Dyslipidemia (no) & 54 & 0.65 & $0.44-0.96$ \\
\hline Renal failure (yes) & 392 & 0.82 & $0.72-0.93$ \\
Renal failure (no) & 104 & 0.71 & $0.54-0.93$ \\
\hline ASA (yes) & 342 & 0.82 & $0.71-0.94$ \\
ASA (no) & 44 & 0.57 & $0.35-0.91$ \\
\hline Anticoagulant (yes) & 402 & 0.81 & $0.72-0.92$ \\
Anticoagulant (no) & 231 & 0.78 & $0.66-0.93$ \\
\hline Comorbidities $\geq 3$ & 215 & 0.81 & $0.68-0.96$ \\
\hline Comorbidities <3 & & &
\end{tabular}

\section{Pork consumption}

\begin{tabular}{|l|c|c|c|}
\hline & $\mathbf{n}$ & SPR & 95\% IC \\
\hline Non-stratified & 446 & 0.77 & $0.66-0.90$ \\
\hline Ischemic heart disease (yes) & 58 & 0.77 & $0.50-1.18$ \\
Ischemic heart disease (no) & 388 & 0.76 & $0.65-0.90$ \\
\hline Obesity (yes) & 114 & 0.75 & $0.54-1.03$ \\
Obesity (no) & 332 & 0.77 & $0.65-0.92$ \\
\hline Diabetes (yes) & 62 & 0.88 & $0.59-1.30$ \\
Diabetes (no) & 384 & 0.75 & $0.63-0.89$ \\
\hline Cerebral vascular & 24 & 0.96 & $0.53-1.74$ \\
disease (yes) & 422 & 0.75 & $0.64-0.88$ \\
Cerebral vascular & & & \\
disease (no) & 184 & 0.78 & $0.61-0.98$ \\
\hline Dyslipidemia (yes) & 262 & 0.77 & $0.63-0.94$ \\
Dyslipidemia (no) & 54 & 0.71 & $0.45-1.13$ \\
\hline Renal failure (yes) & 392 & 0.77 & $0.65-0.91$ \\
\hline Renal failure (no) & 104 & 0.77 & $0.55-1.06$ \\
\hline ASA (yes) & 342 & 0.77 & $0.65-0.91$ \\
\hline ASA (no) & 44 & 0.56 & $0.31-0.99$ \\
\hline Anticoagulant (yes) & 402 & 0.79 & $0.67-0.92$ \\
\hline Anticoagulant (no) & 231 & 0.76 & $0.61-0.95$ \\
\hline Comorbidities $\geq 3$ & & & $0.62-0.96$ \\
\hline Comorbidities <3 & 215 & \\
\hline
\end{tabular}

Sausage meat consumption

\begin{tabular}{|l|c|c|c|}
\hline & $\mathbf{n}$ & SPR & 95\% IC \\
\hline Non-stratified & 446 & 1.05 & $0.94-1.18$ \\
\hline Ischemic heart disease (yes) & 58 & 1.06 & $0.76-1.47$ \\
Ischemic heart disease (no) & 388 & 1.05 & $0.93-1.19$ \\
\hline Obesity (yes) & 114 & 1.10 & $0.86-1.39$ \\
Obesity (no) & 332 & 1.04 & $0.91-1.19$ \\
\hline Diabetes (yes) & 62 & 1.26 & $0.94-1.69$ \\
Diabetes (no) & 384 & 1.02 & $0.90-1.16$ \\
\hline Cerebral vascular & 24 & 0.70 & $0.38-1.30$ \\
disease (yes) & 422 & 1.07 & $0.95-1.21$ \\
Cerebral vascular & & & \\
disease (no) & 184 & 1.03 & $0.85-1.23$ \\
\hline Dyslipidemia (yes) & 262 & 1.07 & $0.92-1.25$ \\
Dyslipidemia (no) & 54 & 0.93 & $0.64-1.33$ \\
\hline Renal failure (yes) & 392 & 1.07 & $0.95-1.21$ \\
Renal failure (no) & 104 & 1.03 & $0.80-1.31$ \\
\hline ASA (yes) & 342 & 1.05 & $0.92-1.20$ \\
\hline ASA (no) & 44 & 0.94 & $0.63-1.41$ \\
\hline Anticoagulant (yes) & 402 & 1.05 & $0.93-1.19$ \\
Anticoagulant (no) & 231 & 1.07 & $0.91-1.26$ \\
\hline Comorbidities $\geq 3$ & 215 & 1.03 & $0.87-1.22$ \\
\hline Comorbidities <3 & & &
\end{tabular}

\section{Fish consumption}

\begin{tabular}{|l|c|c|c|}
\hline & $\mathrm{n}$ & SPR & $95 \%$ IC \\
\hline Non-stratified & 446 & 0.98 & $0.87-1.11$ \\
\hline Ischemic heart disease (yes) & 58 & 1.24 & $0.92-1.67$ \\
Ischemic heart disease (no) & 388 & 0.95 & $0.83-1.08$ \\
\hline Obesity (yes) & 114 & 0.98 & $0.77-1.26$ \\
Obesity (no) & 332 & 0.98 & $0.85-1.12$ \\
\hline Diabetes (yes) & 62 & 0.93 & $0.67-1.29$ \\
Diabetes (no) & 384 & 0.99 & $0.87-1.13$ \\
\hline Cerebral vascular & 24 & 1.14 & $0.71-1.84$ \\
disease (yes) & 422 & 0.98 & $0.86-1.10$ \\
Cerebral vascular & & & \\
disease (no) & 184 & 1.00 & $0.83-1.20$ \\
\hline Dyslipidemia (yes) & 262 & 0.97 & $0.83-1.14$ \\
\hline Dyslipidemia (no) & 54 & 0.96 & $0.67-1.36$ \\
\hline Renal failure (yes) & 392 & 0.99 & $0.87-1.12$ \\
Renal failure (no) & 104 & 1.09 & $0.86-1.38$ \\
\hline ASA (yes) & 342 & 0.96 & $0.83-1.10$ \\
\hline ASA (no) & 44 & 1.00 & $0.68-1.47$ \\
\hline Anticoagulant (yes) & 231 & 0.98 & $0.83-1.16$ \\
Anticoagulant (no) & & 0.98 & $0.83-1.17$ \\
\hline Comorbidities $\geq 3$ & & \\
Comorbidities <3 & 215 & 0.99 & $0.87-12$ \\
\hline
\end{tabular}


Vegetable consumption

\begin{tabular}{|l|c|c|c|}
\hline & $\mathbf{n}$ & SPR & 95\% IC \\
\hline Non-stratified & 446 & 0.99 & $0.87-1.12$ \\
\hline Ischemic heart disease (yes) & 58 & 1.07 & $0.77-1.48$ \\
Ischemic heart disease (no) & 388 & 0.98 & $0.86-1.12$ \\
\hline Obesity (yes) & 114 & 1.04 & $0.80-1.34$ \\
Obesity (no) & 332 & 0.99 & $0.86-1.13$ \\
\hline Diabetes (yes) & 62 & 1.10 & $0.80-1.51$ \\
Diabetes (no) & 384 & 0.97 & $0.85-1.12$ \\
\hline Cerebral vascular & 24 & 1.13 & $0.69-1.84$ \\
disease (yes) & & & \\
Cerebral vascular & 422 & 0.98 & $0.87-1.12$ \\
disease (no) & & & \\
\hline Dyslipidemia (yes) & 184 & 1.03 & $0.85-1.24$ \\
Dyslipidemia (no) & 262 & 0.96 & $0.82-1.14$ \\
\hline Renal failure (yes) & 54 & 1.05 & $0.75-1.47$ \\
Renal failure (no) & 392 & 0.99 & $0.86-1.13$ \\
\hline ASA (yes) & 104 & 1.02 & $0.79-1.32$ \\
ASA (no) & 342 & 0.98 & $0.85-1.12$ \\
\hline Anticoagulant (yes) & 44 & 0.95 & $0.64-1.41$ \\
Anticoagulant (no) & 402 & 0.99 & $0.87-1.13$ \\
\hline Comorbidities $\geq 3$ & 231 & 1.05 & $0.89-1.24$ \\
Comorbidities <3 & 215 & 0.93 & $0.77-1.12$ \\
\hline
\end{tabular}

\section{Milk consumption}

\begin{tabular}{|l|c|c|c|}
\hline & $\mathbf{n}$ & SPR & 95\% IC \\
\hline Non-stratified & 446 & 0.81 & $0.71-0.94$ \\
\hline Ischemic heart disease (yes) & 58 & 0.88 & $0.62-1.26$ \\
Ischemic heart disease (no) & 388 & 0.81 & $0.69-0.94$ \\
\hline Obesity (yes) & 114 & 0.67 & $0.48-0.92$ \\
Obesity (no) & 332 & 0.85 & $0.73-0.99$ \\
\hline Diabetes (yes) & 62 & 0.97 & $0.69-1.37$ \\
Diabetes (no) & 384 & 0.79 & $0.67-0.92$ \\
\hline Cerebral vascular & 24 & 0.93 & $0.54-1.60$ \\
disease (yes) & 422 & 0.81 & $0.70-0.93$ \\
Cerebral vascular & & & \\
disease (no) & 184 & 0.75 & $0.60-0.94$ \\
\hline Dyslipidemia (yes) & 262 & 0.87 & $0.73-1.04$ \\
Dyslipidemia (no) & 54 & 1.01 & $0.71-1.43$ \\
\hline Renal failure (yes) & 392 & 0.78 & $0.67-0.91$ \\
Renal failure (no) & 104 & 0.81 & $0.61-1.08$ \\
\hline ASA (yes) & 342 & 0.82 & $0.71-0.96$ \\
ASA (no) & 44 & 1.08 & $0.73-1.59$ \\
\hline Anticoagulant (yes) & 215 & 0.79 & $0.68-0.91$ \\
Anticoagulant (no) & & 0.86 & $0.71-1.04$ \\
\hline Comorbidities $\geq 3$ & 231 & $0.62-0.94$ \\
\hline Comorbidities <3 & & \\
\hline
\end{tabular}

Carbohydrate intake

\begin{tabular}{|l|c|c|c|}
\hline & $\mathbf{n}$ & SPR & 95\% IC \\
\hline Non-stratified & 446 & 1.00 & $0.91-1.11$ \\
\hline Ischemic heart disease (yes) & 58 & 1.03 & $0.79-1.34$ \\
Ischemic heart disease (no) & 388 & 1.00 & $0.90-1.11$ \\
\hline Obesity (yes) & 114 & 1.00 & $0.81-1.23$ \\
Obesity (no) & 332 & 1.00 & $0.89-1.12$ \\
\hline Diabetes (yes) & 62 & 0.98 & $0.74-1.28$ \\
Diabetes (no) & 384 & 1.00 & $0.91-1.12$ \\
\hline Cerebral vascular & 24 & 1.10 & $0.73-1.64$ \\
disease (yes) & 422 & 1.00 & $0.90-1.11$ \\
Cerebral vascular & & & \\
disease (no) & 184 & 1.01 & $0.85-1.17$ \\
\hline Dyslipidemia (yes) & 262 & 1.00 & $0.88-1.14$ \\
Dyslipidemia (no) & 54 & 1.06 & $0.81-1.39$ \\
\hline Renal failure (yes) & 392 & 1.00 & $0.90-1.11$ \\
Renal failure (no) & 104 & 1.01 & $0.82-1.24$ \\
\hline ASA (yes) & 342 & 1.00 & $0.89-1.12$ \\
\hline ASA (no) & 44 & 1.04 & $0.76-1.41$ \\
\hline Anticoagulant (yes) & 402 & 1.00 & $0.90-1.11$ \\
Anticoagulant (no) & 231 & 1.01 & $0.88-1.16$ \\
\hline Comorbidities $\geq 3$ & 215 & 0.99 & $0.86-1.15$ \\
\hline Comorbidities <3 & & &
\end{tabular}

\section{Dairy products consumption}

\begin{tabular}{|l|c|c|c|}
\hline & $\mathbf{n}$ & SPR & 95\% IC \\
\hline Non-stratified & 446 & 1.01 & $0.83-1.21$ \\
\hline Ischemic heart disease (yes) & 58 & 1.36 & $0.88-2.11$ \\
Ischemic heart disease (no) & 388 & 0.94 & $0.77-1.16$ \\
\hline Obesity (yes) & 114 & 0.93 & $0.62-1.40$ \\
Obesity (no) & 332 & 1.03 & $0.83-1.27$ \\
\hline Diabetes (yes) & 62 & 0.85 & $0.49-1.46$ \\
Diabetes (no) & 384 & 1.03 & $0.84-1.26$ \\
\hline Cerebral vascular & 24 & 1.35 & $0.68-2.71$ \\
disease (yes) & 422 & 0.98 & $0.81-1.20$ \\
Cerebral vascular & & & \\
disease (no) & 184 & 0.95 & $0.70-1.27$ \\
\hline Dyslipidemia (yes) & 262 & 1.07 & $0.84-1.36$ \\
Dyslipidemia (no) & 54 & 1.32 & $0.83-2.09$ \\
\hline Renal failure (yes) & 392 & 0.96 & $0.78-1.17$ \\
Renal failure (no) & 104 & 0.89 & $0.59-1.35$ \\
\hline ASA (yes) & 342 & 1.06 & $0.87-1.30$ \\
\hline ASA (no) & 44 & 1.06 & $0.59-1.91$ \\
\hline Anticoagulant (yes) & 402 & 1.00 & $0.82-1.22$ \\
Anticoagulant (no) & 231 & 1.02 & $0.79-1.31$ \\
\hline Comorbidities $\geq 3$ & 215 & 0.99 & $0.75-1.31$ \\
\hline Comorbidities <3 & & & \\
\hline
\end{tabular}


Fruit consumption

\begin{tabular}{|l|c|c|c|}
\hline & $\mathbf{n}$ & SPR & $95 \%$ IC \\
\hline Non-stratified & 446 & 1.00 & $0.90-1.11$ \\
\hline Ischemic heart disease (yes) & 58 & 0.97 & $0.74-1.28$ \\
Ischemic heart disease (no) & 388 & 1.00 & $0.90-1.12$ \\
\hline Obesity (yes) & 114 & 0.97 & $0.78-1.21$ \\
Obesity (no) & 332 & 1.01 & $0.89-1.13$ \\
\hline Diabetes (yes) & 62 & 1.02 & $0.78-1.34$ \\
Diabetes (no) & 384 & 1.00 & $0.89-1.12$ \\
\hline Cerebral vascular & 24 & 0.96 & $0.62-1.48$ \\
disease (yes) & 422 & 1.00 & $0.90-1.11$ \\
Cerebral vascular & & & \\
disease (no) & 184 & 0.98 & $0.84-1.15$ \\
\hline Dyslipidemia (yes) & 262 & 1.02 & $0.89-1.16$ \\
Dyslipidemia (no) & 54 & 1.06 & $0.80-1.40$ \\
\hline Renal failure (yes) & 392 & 0.99 & $0.89-1.11$ \\
Renal failure (no) & 104 & 1.03 & $0.84-1.27$ \\
\hline ASA (yes) & 342 & 0.99 & $0.88-1.12$ \\
ASA (no) & 44 & 1.06 & $0.78-1.45$ \\
\hline Anticoagulant (yes) & 402 & 0.99 & $0.89-1.10$ \\
Anticoagulant (no) & 231 & 1.02 & $0.88-1.17$ \\
\hline Comorbidities $\geq 3$ & 215 & 0.98 & $0.84-1.14$ \\
\hline Comorbidities <3 & & &
\end{tabular}

\section{Beer consumption}

\begin{tabular}{|l|c|c|c|}
\hline & $\mathbf{n}$ & SPR & 95\% IC \\
\hline Non-stratified & 446 & 2.21 & $1.68-2.90$ \\
\hline Ischemic heart disease (yes) & 58 & 2.77 & $1.36-5.62$ \\
Ischemic heart disease (no) & 388 & 2.11 & $1.57-2.83$ \\
\hline Obesity (yes) & 114 & 3.11 & $1.98-4.87$ \\
Obesity (no) & 332 & 1.86 & $1.32-2.62$ \\
\hline Diabetes (yes) & 62 & 1.88 & $0.79-7.59$ \\
Diabetes (no) & 384 & 2.25 & $1.69-3.00$ \\
\hline Cerebral vascular & 24 & 1.95 & $0.50-6.09$ \\
disease (yes) & 422 & 2.21 & $1.68-2.92$ \\
Cerebral vascular & & & \\
disease (no) & 184 & 2.07 & $1.33-3.21$ \\
\hline Dyslipidemia (yes) & 262 & 2.38 & $1.70-3.33$ \\
\hline Dyslipidemia (no) & 54 & 2.36 & $1.01-5.52$ \\
\hline Renal failure (yes) & 392 & 2.17 & $1.63-2.90$ \\
Renal failure (no) & 104 & 0.90 & $0.34-2.40$ \\
\hline ASA (yes) & 342 & 2.45 & $1.85-3.24$ \\
\hline ASA (no) & 44 & 1.94 & $0.74-5.09$ \\
\hline Anticoagulant (yes) & 402 & 2.18 & $1.64-2.89$ \\
\hline Anticoagulant (no) & 231 & 2.33 & $1.55-3.49$ \\
\hline Comorbidities $\geq 3$ & 215 & 2.12 & $1.46-3.06$ \\
\hline Comorbidities <3 & & & \\
\hline
\end{tabular}

Wine consumption

\begin{tabular}{|l|c|c|c|}
\hline & $\mathbf{n}$ & SPR & 95\% IC \\
\hline Non-stratified & 446 & 1.85 & $1.48-2.32$ \\
\hline Ischemic heart disease (yes) & 58 & 1.62 & $0.84-3.09$ \\
Ischemic heart disease (no) & 388 & 1.88 & $1.47-2.39$ \\
\hline Obesity (yes) & 114 & 2.26 & $1.47-3.46$ \\
Obesity (no) & 332 & 1.78 & $1.37-2.31$ \\
\hline Diabetes (yes) & 62 & 0.90 & $0.37-2.16$ \\
Diabetes (no) & 384 & 2.00 & $1.59-2.53$ \\
\hline Cerebral vascular & 24 & 3.01 & $1.49-6.09$ \\
disease (yes) & 422 & 1.77 & $1.40-2.25$ \\
Cerebral vascular & & & \\
disease (no) & 184 & 2.42 & $1.80-3.25$ \\
\hline Dyslipidemia (yes) & 262 & 1.48 & $1.06-2.07$ \\
Dyslipidemia (no) & 54 & 1.55 & $0.78-3.09$ \\
\hline Renal failure (yes) & 392 & 1.91 & $1.51-2.43$ \\
Renal failure (no) & 104 & 1.37 & $0.80-2.37$ \\
\hline ASA (yes) & 342 & 1.99 & $1.56-2.54$ \\
\hline ASA (no) & 44 & 1.69 & $0.81-3.51$ \\
\hline Anticoagulant (yes) & 402 & 1.87 & $1.48-2.37$ \\
Anticoagulant (no) & 231 & 1.56 & $1.10-2.20$ \\
\hline Comorbidities $\geq 3$ & 215 & 2.17 & $1.61-2.92$ \\
\hline Comorbidities <3 & & &
\end{tabular}

\section{Spirit consumption}

\begin{tabular}{|l|c|c|c|}
\hline & $\mathbf{n}$ & SPR & 95\% IC \\
\hline Non-stratified & 446 & 0.57 & $0.46-0.72$ \\
\hline Ischemic heart disease (yes) & 58 & 0.55 & $0.28-1.10$ \\
Ischemic heart disease (no) & 388 & 0.58 & $0.46-0.74$ \\
\hline Obesity (yes) & 114 & 0.39 & $0.23-0.68$ \\
Obesity (no) & 332 & 0.64 & $0.50-0.82$ \\
\hline Diabetes (yes) & 62 & 0.63 & $0.34-1.17$ \\
Diabetes (no) & 384 & 0.56 & $0.44-0.72$ \\
\hline Cerebral vascular & 24 & 0.67 & $0.25-1.76$ \\
disease (yes) & 422 & 0.57 & $0.45-0.72$ \\
Cerebral vascular & & & \\
disease (no) & 184 & 0.59 & $0.42-0.83$ \\
\hline Dyslipidemia (yes) & 262 & 0.56 & $0.42-0.76$ \\
Dyslipidemia (no) & 54 & 0.55 & $0.27-1.15$ \\
\hline Renal failure (yes) & 392 & 0.57 & $0.45-0.73$ \\
Renal failure (no) & 104 & 0.34 & $0.18-0.63$ \\
\hline ASA (yes) & 342 & 0.62 & $0.49-0.79$ \\
ASA (no) & 44 & 0.91 & $0.49-1.69$ \\
\hline Anticoagulant (yes) & 402 & 0.53 & $0.42-0.68$ \\
\hline Anticoagulant (no) & 231 & 0.56 & $0.40-0.79$ \\
\hline Comorbidities $\geq 3$ & 215 & 0.58 & $0.43-0.79$ \\
\hline Comorbidities <3 & & & \\
\hline
\end{tabular}


Employment rate

\begin{tabular}{|l|c|c|c|}
\hline & $\mathbf{n}$ & SPR & 95\% IC \\
\hline Non-stratified & 446 & 0.47 & $0.40-0.56$ \\
\hline Ischemic heart disease (yes) & 58 & 0.24 & $0.13-0.43$ \\
Ischemic heart disease (no) & 388 & 0.51 & $0.43-0.61$ \\
\hline Obesity (yes) & 114 & 0.62 & $0.45-0.85$ \\
Obesity (no) & 332 & 0.44 & $0.36-0.53$ \\
\hline Diabetes (yes) & 62 & 0.24 & $0.13-0.44$ \\
Diabetes (no) & 384 & 0.51 & $0.43-0.61$ \\
\hline Cerebral vascular & 24 & 0.06 & $0.01-0.28$ \\
disease (yes) & & & \\
Cerebral vascular & 422 & 0.50 & $0.42-0.59$ \\
disease (no) & & & \\
\hline Dyslipidemia (yes) & 184 & 0.42 & $0.32-0.55$ \\
Dyslipidemia (no) & 262 & 0.51 & $0.42-0.64$ \\
\hline Renal failure (yes) & 54 & 0.16 & $0.08-0.33$ \\
Renal failure (no) & 392 & 0.53 & $0.44-0.62$ \\
\hline ASA (yes) & 104 & 0.35 & $0.23-0.51$ \\
ASA (no) & 342 & 0.52 & $0.44-0.63$ \\
\hline Anticoagulant (yes) & 44 & 0.27 & $0.14-0.52$ \\
Anticoagulant (no) & 402 & 0.50 & $0.43-0.60$ \\
\hline Comorbidities $\geq 3$ & 231 & 0.30 & $0.23-0.40$ \\
Comorbidities <3 & 215 & 0.67 & $0.54-0.82$ \\
\hline
\end{tabular}

Low education

\begin{tabular}{|l|c|c|c|}
\hline & $\mathbf{n}$ & SPR & 95\% IC \\
\hline Non-stratified & 446 & 1.71 & $1.45-2.00$ \\
\hline Ischemic heart disease (yes) & 58 & 2.13 & $1.46-3.10$ \\
Ischemic heart disease (no) & 388 & 1.64 & $1.37-1.95$ \\
\hline Obesity (yes) & 114 & 1.89 & $1.38-2.60$ \\
Obesity (no) & 332 & 1.63 & $1.35-1.96$ \\
\hline Diabetes (yes) & 62 & 2.37 & $1.67-3.38$ \\
Diabetes (no) & 384 & 1.59 & $1.33-1.91$ \\
\hline Cerebral vascular & 24 & 2.62 & $1.55-4.42$ \\
disease (yes) & 422 & 1.65 & $1.39-1.95$ \\
Cerebral vascular & & & \\
disease (no) & 184 & 1.89 & $1.50-2.38$ \\
\hline Dyslipidemia (yes) & 262 & 1.56 & $1.27-1.95$ \\
Dyslipidemia (no) & 54 & 1.91 & $1.26-2.91$ \\
\hline Renal failure (yes) & 392 & 1.67 & $1.40-1.98$ \\
\hline Renal failure (no) & 104 & 2.23 & $1.68-2.96$ \\
\hline ASA (yes) & 342 & 1.53 & $1.27-1.85$ \\
\hline ASA (no) & 44 & 2.02 & $1.27-3.22$ \\
\hline Anticoagulant (yes) & 402 & 1.67 & $1.41-1.97$ \\
Anticoagulant (no) & 231 & 2.25 & $1.87-2.72$ \\
\hline Comorbidities $\geq 3$ & 215 & 1.08 & $0.81-1.46$ \\
\hline Comorbidities <3 & & &
\end{tabular}

disease or diabetes, accordingly to reported data about a possible association of gout with features of the metabolic syndrome. We do not know if patients have changed their weight after gout diagnosis, but our data show that a high proportion of subjects still haven't gained a healthy physical status. On the contrary, in our study sample we observed a prevalence of smokers lower than the expected. The role of smoking as a risk factor for gout is still debated because of controversial results from epidemiological studies evaluating the relationship between smoking and serum uric acid levels or gout incidence $(11,12)$. However, smoking cessation is recommended in current guidelines for gout management (4). Stratified analyses identified obesity, ischemic heart disease and related therapies as possible confounders, being associated with a significant lower prevalence of smoking. Therefore, a possible explanation for our study should be that a number of subjects have been forced to reduce smoking because of the associated diseases.
Alcohol consumption is a well-recognized risk factor for hyperuricemia and gout (1315). Recent studies have clarified that the impact on the risk of gout varies depending on the type and amount of alcoholic beverage consumed (14). A moderate consumption of wine seems not to increase the risk of gout, while consumption of beer and hard liquor is associated with increased risk of incident gout at any amount. Furthermore, beer is known to have an alcohol-independent effect on serum urate concentration related to its high content of guanosine. Relying on those findings, we chose a different cut-off for defining a high consumption of wine, beer and spirits. Our patients showed a higher consumption of wine and beer compared to the general population, but a lower consumption of spirits. This point reflect a still inadequate awareness of the impact of alcoholic beverages on gout, so that only the intake of drinks with a higher content of alcohol is reduced in our patients, but the overall alcohol intake is still higher than the expected. 
A high red meat and seafood intake has been extensively reported to be associated with hyperuricemia and an increased risk of gout, and a diet characterized by a low purine intake is one of the best-known dietary interventions for gout (16). On this basis, the finding of a lower consumption of red meat in our study population suggests that reducing red meat intake is widely stressed among gouty patients, even if recent approaches prefer not to suggest a rigid purine restricted diet (17). However, among our patients we did not find a higher consumption of carbohydrates as a compensation for the reduced intake of meat. The role of milk and dairy products on the risk of gout has only recently been investigated. A higher consumption of low-fat dairy products have been related to a lower incidence of gout (16) and specific milk components have been studied as possible determinants of the protective effect of milk on gout flares (18). In our study population we observed a lower consumption of milk, suggesting that the role of milk and derivates in dietary intervention for gout has not adequately underlined yet. Only in patients affected by renal failure we found a higher intake of milk and dairy foods, as a possible compensation for a rigid purine restricted diet.

We did not find any significant difference from the general population concerning fruit and vegetables consumption. At present, even if the negative effect of fructose on hyperuricemia and gout is well established for sweetened beverages $(19,20)$, the role of fruit consumption is still debated because of contradictory results from different studies (21). Conversely, vegetables consumption is recommended as it seems to reduce the risk of gout $(16,17)$.

Lastly, we observed a lower employment rate and a lower level of education in patients with gout. Even if gout was known in the past as the kings' disease because of its high prevalence among men with a higher socio-economic status, our finding suggests that gout should no longer be considered peculiar of the higher social classes. This finding could reflect significant changes in dietary and lifestyle habits in the general population (22). Moreover, results from stratified analysis suggest that the presence of comorbidities should significantly account for the lower employment rate, especially in relationship with more disabling diseases such as stroke or renal failure.

\section{CONCLUSIONS}

In conclusion, our results suggest that gouty patients' lifestyle is still partially different from the recommended. There is a good awareness of some traditional risk factors for gout, such as a purine-rich diet or hard liquor consumption, but many other dietary and lifestyle interventions are still far from an optimal enforcement. The presence of comorbidities could also significantly affect patients' behaviour. Further efforts should be made to sensitize gouty patients to the importance of lifestyles modification.

\section{REFERENCES}

1. Brook RA, Forsythe A, Smeeding JE, Lawrence Edwards N. Chronic gout: epidemiology, disease progression, treatment and disease burden. Curr Med Res Opin. 2010; 26: 2813-21.

2. Singh JA, Reddy SG, Kundukulam J. Risk factors for gout and prevention: a systematic review of the literature. Curr Opin Rheumatol. 2011; 23: 192-202.

3. Zhang W, Doherty M, Bardin T, et al. EULAR evidence based recommendations for gout. Part II: Management. Report of a task force of the EULAR Standing Committee for International Clinical Studies Including Therapeutics (ESCISIT). Ann Rheum Dis. 2006; 65: 131224.

4. Khanna D, Fitzgerald JD, Khanna PP, et al. American College of Rheumatology guidelines for management of gout. Part 1: systematic nonpharmacologic and pharmacologic therapeutic approaches to hyperuricemia. Arthritis Care Res (Hoboken). 2012; 64: 143146.

5. Roddy E, Zhang W, Doherty M. Concordance of the management of chronic gout in a UK primary-care population with the EULAR gout recommendations. Ann Rheum Dis. 2007; 66: 1311-5.

6. Shulten P, Thomas J, Miller M, et al. The role of diet in the management of gout: a comparison of knowledge and attitudes to current evidence. J Human Nutr Diet. 2009; 22: 3-11. 
7. Loghi M, D’Errico A. Health for All - Italia, an informative health system. Ital J Public Health. 2008; 5: 107-111.

8. Rothman KJ, Greenland S, Lash TL. Modern epidemiology, 3rd ed. Philadelphia, PA: Lippincot Williams \& Wilkins. 2008; 67-69.

9. Choi HK, Atkinson K, Karlson EW, Curhan G. Obesity, weight change, hypertension, diuretic use, and risk of gout in men: the health professionals follow-up study. Arch Intern Med. 2005; 165: 742-8.

10. Williams PT. Effects of diet, physical activity and performance, and body weight on incident gout in ostensibly healthy, vigorously active men. Am J Clin Nutr. 2008; 87: 1480-7.

11. Hanna BE, Hamed JM, Touhala LM. Serum uric Acid in smokers. Oman Med J. 2008; 23: 269-74.

12. Wang W, Krishnan E. Cigarette smoking is associated with a reduction in the risk of incident gout: results from the Framingham Heart Study original cohort. Rheumatology (Oxford). 2015; 54: 91-5.

13. Bhole V, de Vera M, Rahman MM, et al. Epidemiology of gout in women: Fifty-two-year followup of a prospective cohort. Arthritis Rheum. 2010; 62: 1069-76.

14. Choi HK, Atkinson K, Karlson EW, et al. Alcohol intake and risk of incident gout in men: a prospective study. Lancet. 2004; 363: 1277 81.

15. Rothenbacher D, Primatesta P, Ferreira A, et al. Frequency and risk factors of gout flares in a large population-based cohort of incident gout. Rheumatology (Oxford). 2011; 50: 97381.

16. Choi HK, Atkinson $\mathrm{K}$, Karlson EW, et al. $\mathrm{Pu}-$ rine-rich foods, dairy and protein intake, and the risk of gout in men. N Engl J Med. 2004; 350: 1093-103.

17. Choi HK. A prescription for lifestyle change in patients with hyperuricemia and gout. Curr Opin Rheumatol. 2010; 22: 165-72.

18. Dalbeth N, Ames R, Gamble GD, et al. Effects of skim milk powder enriched with glycomacropeptide and G600 milk fat extract on frequency of gout flares: a proof-of-concept randomised controlled trial. Ann Rheum Dis. 2012; 71: 929-34.

19. Choi HK, Curhan G. Soft drinks, fructose consumption, and the risk of gout in men: prospective cohort study. BMJ 2008; 336: 309-12.

20. Choi HK, Willett W, Curhan G. Fructose-rich beverages and risk of gout in women. JAMA 2010; 304: 2270-8.

21. Sun SZ, Flickinger BD, Williamson-Hughes PS, Empie MW. Lack of association between dietary fructose and hyperuricemia risk in adults. Nutr Metab (Lond). 2010; 7: 16.

22. Saag KG, Choi H. Epidemiology, risk factors, and lifestyle modifications for gout. Arthritis Res Ther. 2006; 8: S2.

\section{ACKNOWLEDGMENTS}

The Authors acknowledge the KING study group: Alarico Ariani, Department of Rheumatology, Polytechnic University of Marche, Ancona, Italy; Alessandra Galossi, UOC Medicina Interna ad indirizzo Epato-Gastroenterologico, AO S. Giuseppe, Marino (RM); Ciro Lauriti, Rheumatology Unit, Ospedale Civile Pescara, (PE); Elena Fracassi and Luca Idolazzi, Rheumatology Unit, Azienda Integrata Ospedale - Università di Verona, (VR); Marco Bardelli and Enrico Selvi, Rheumatology Unit, Policlinico 'Le Scotte', Siena (SI); Enrico Tirri, Dipartimento di Scienze Mediche - UOSD di Reumatologia, Ospedale San Giovanni Bosco, Napoli (NA); Federica Furini, Rheumatology Unit, Department of Medical Sciences, University of Ferrara, Azienda Ospedaliero - Universitaria S. Anna, Ferrara (FE); Flora Inverardi, Chair and Unit of Rheumatology, IRCCS Fondazione Policlinico San Matteo - Università di Pavia, (PV); Andrea Calabrò and Francesco Porta, Dipartimento di Medicina Clinica e Sperimentale, SOD Reumatologia, AOUC, University of Firenze, (FI); Raffaele Bittelli and Francesco Venturino, Rheumatology Unit, Casa di Cura Reumatologica Oliveti Cotronei (KR); Franco Capsoni, Unità Operativa Reumatologia Universitaria, Istituto Ortopedico Galeazzi - Università di Milano, (MI); Immacolata Prevete and Giandomenico Sebastiani, Rheumatology Unit, Azienda Ospedaliera San Camillo - Forlanini, Roma (RM); Carlo Selmi and Gianluigi Fabbriciani, Division of Rheumatology and Clinical Immunology, Humanitas Clinical and Research Center, Rozzano (MI); Giovanni D'Avola, Rheumatology Unit, ASP3 Catania, (CT); Giulia Botticella, Clinica Reumatologica Dipartimento di Medicina Interna e Specialità Mediche, University of Genova, (GE); Francesca Serale and Giulia Seminara, Rheumatology Unit, ASO $S$ Croce e Carle Cuneo (CN); Giuseppe D'Alessandro, SS di Malattie Apparato Osteoarticolare ed Immunoproliferative, Azienda Ospedaliero-Universitaria 'Ospedali Riuniti' di Foggia, (FG); Leonardo Santo, Rheumatology Unit, ASL BT - DSS 
4 Barletta, (BT); Lorena Longato, Ambulatorio di Reumatologia, Poliambulatorio ASL, ASL Biella, (BI); Eleonora Zaccara and Luigi Sinigaglia, Struttura Complessa di Reumatologia DH, Istituto Ortopedico G. Pini, Milano (MI); Marco Atteritano, Unit of Rheumatology, Department of Clinical and Experimental Medicine University of Messina, (ME); Marco Broggini, SSD di Reumatologia, A.O. Ospedale di Circolo e Fondazione Macchi, Varese (VA); Marta Caprioli, UO di Medicina Interna, Istituto Clinico Beato Matteo, Vigevano $(P V)$; Marta Favero, Rheumatology Unit,
Department of Medicine - DIMED, University Hospital of Padova, (PD); Salvatore Salli, Rheumatology Unit, Policlinico Universitario Paolo Giaccone, Palermo (PA); Marco Scarati and Simone Parisi, Rheumatology Unit A.O. Città della Salute e della Scienza di Torino, (TO); Nazzarena Malavolta and Stefania Corvaglia, Rheumatology Unit, Azienda Ospedaliera Universitaria di Bologna - Policlinico S. Orsola Malpighi, Bologna (BO); Salvatore Scarpato and Vittorio Veneto, Rheumatology Unit, Ospedale 'M. Scarlato' ASL Salerno, Scafati (SA). 